EUROPEAN ASSOCIATION

FOR THE STUDY OF DIABETES
ASSOCIATION EUROPEENNE

POUR L'ÉTUDE

DU DIABETE
EUROPÄISCHE

GESELLSCHAFT FUR

DIABETOLOGIE

\title{
ORGANIZATION SECTION
}

President: W. CRedTzFeldi, Göttingen (retires 1974)

Vice Presidents: B. Helcman, Umeå (retires 1973) J. VAGUE, Marseille (retires 1975)

Past President: K. Lundbaek, Aarhus
Secretary: V. CONARD, Bruxelles (retires 1973)

Treasurer: $\quad$ R.F. MAHLER, Cardiff (retires 1975)

Editors-in-Chief of Diabetologia

W. CReutzFedDT, Göttingen (retires 1976)

K. SaHöffLING, Frankfurt (retires 1976)

The administrative offices of the Association are located with the

Executive Director, Mr. J.G.L. Jackson, 3/6 Alfred Place, London WC 1E 7EE, England.

\section{COUNCIL}

Term expiring 1973

J.G. Aurvisatos, Athens

A. Beringer, Vienna

G. Pozza, Milan

J.L. Rodriguez-Mrñón, Madrid
Term expiring 1974

K. D. HæPP, Munich

P. LEFìBVRE, Liège

D. A. FYKE, London

W. Stauffacher, Geneva
Term expiring 1975

V. ANDReant, Rome

H. KEEN, London

J. Jervel, Oslo

Z. Skrabalo, Zagreb

\section{HONORARY MEMBERS}

C.H. Best, Toronto - M. Derot, Paris - R. Levine, Duarte - Sir Frank Young, Cambridge

\section{SUPPORTING AND ASSOCIATE MEMBERS}

Ames Europe, Slough, Buckinghamshire, Aron of Suresnes, France - Boehringer, Mannheim, Germany - British Insulin Manufacturers, Great Britain - Chemie Grünenthal, Stolberg - Eli Lilly, Rome, Italy - Hoechst, Frankfurt - HoffmannLa Roche, Basel - Hormon Chemie, München - Novo Foundation, Copenhagen - Pfizer Europe, Bruxelles - Phartec, France Rona, Hitchin, Hertsfordshire - Sandoz A.G., Basel - Servier, Neuilly-sur-Seine - Upjohn Co., Kalamazoo - Winthrop Laboratories, Surbiton-on-Thames, Surrey.

\section{Förderpreis der Deutschen Diabetes-Gesellschaft}

Für hervorragende Dissertationen auf dem Gebiet der Diabetologie wurde von den Firmen Bayer und Schering AG der ,Förderpreis der Deutschen Diabetes-Gesellschaft" gestiftet. Er soll angehende Wissenschaftler auszeichnen und ihnen ermöglichen, sich an theoretischen Instituten des In- und Auslandes fortzubilden. Der Preis, der von der Deutschen Diabetes-Gesellschaft mit einer Urkunde verliehen wird, ist mit DM 11000.- dotiert, davon sind DM 10000. - für ein Stipendium bestimmt.

Deutschsprachige Bewerber werden gebeten, ihre Arbeit direkt mit der Promotionsurkunde oder - im Falle eines noch nicht abgeschlossenen Promotionsverfahrens - über den Doktorvater mit dem Referat über die Arbeit und einem Lebenslauf an den Vorsitzenden der Deutschen Diabetes-Gesellschaft, Herrn Prof. Dr. H. Mehnert, III. Med. Abt. des Krankenhauses München-Schwabing. 8000 München 23, Kölner Platz 1, bis spätesten 1. 3. 1973 einzureichen. Die Dissertation soll nicht älter als zwei Jahre sein und die Note ,sehr gut" erhalten haben. Mit dem Preis können auch solche in deutscher Sprache verfaßBten Arbeiten ausgezeichnet werden, die den Dissertationen der deutschen Hochschulen entsprechen und an deutschsprachige Universitäten eingereicht wurden.

Die Verleihung erfölgt anläßlich des 8. Kongresses der Deutschen Diabetes-Gesellschaft in München (21.-23.6. 1973).

\section{Ferdinand-Bertram-Preis}

Der „Ferdinand-Bertram-Preis “ wurde von der Firma Boehringer Mannheim GmbH für hervorragende und wegweisende wissenschaftliche Arbeiten auf dem. Gebiet der Diabetologie gestiftet. Er wird von der Deutschen-Diabetes-Gesellschaft verliehen und ist mit DM 5000 . - dotiert.

Deutschsprachige Bewerber, die das 40. Lebensjahr nicht übersehritten haben, werden gebeten, ihre wissenschaftlichen Arbeiten an den Vorsitzenden der Deutsehen Diabetes-Gesellschaft, Prof. Dr. H. Mehnert, III. Med. Abt. des Krankenhauses München-Schwabing, 8000 Mün- chen 23, Kölner Platz 1, unter Angabe von Anschrift und Lebenslauf bis spätestens 1. 3. 1973 einzusenden. Die Ar. beiten sollten innerhalb des deutschen Sprachraumes entstanden sein. Der Lebenslauf soll den wissenschaftlichen Werdegang enthalten.

Die Verleihung des Preises erfolgt zu Beginn der 8. Tagung der Deutschen Diabetes-Gesellschaft in München (21.-23. 6. 1973). Der Preistrëger soll bei dieser Gelegenheit eine Bertram-Preis-Vorlesung halten, die über sein spezielles Arbeitsgebiet berichtet. 